\title{
Power Spectra of Ionospheric Scintillations
}

\section{George Jandieri ${ }^{1}$, Akira Ishimaru ${ }^{2}$, Banmali Rawat ${ }^{3}$, Oleg Kharshiladze ${ }^{4}$, and Zhuzhuna Diasamidze ${ }^{5}$}

\author{
${ }^{1}$ Institute of Cybernetics, Georgian Technical University, Tbilisi, Georgia \\ ${ }^{2}$ Department of Electrical Engineering, University of Washington, Seattle, USA \\ ${ }^{3}$ Department of Electrical and Biomedical Engineering, University of Nevada, Reno, USA \\ ${ }^{4}$ Tbilisi State University, Tbilisi, Georgia \\ ${ }^{5}$ Batumi Shota Rustaveli State University, Batumi, Georgia \\ *corresponding author, E-mail: georgejandieri7@gmail.com
}

\begin{abstract}
Second order statistical moments of the phase fluctuations are obtained taking into account the boundary condition, diffraction effects and polarization coefficients of the ordinary and extraordinary waves. The variance and the correlation function are calculated for arbitrary 3D spectral function of electron density fluctuations containing both anisotropic Gaussian and power-law spectra; anisotropy coefficient and the orientation angle of elongated plasma irregularities. The phase scintillation index and the scintillation level are analyzed numerically. Maximum of the scintillation index for small-scale irregularities is in the interval 0.2-0.3 corresponding to the moderate scintillation intensity, within the weak-scatter regime. Splashes are revealed for different anisotropy factor of elongated largescale irregularities varying orientation angle with respect to the lines of force of geomagnetic field. Scintillation index is calculated for small-scale irregularities using the "frozenin" assumption and taking into account movement of rigid irregularities. Log-log plots of the power spectrum of the intensity fluctuations have the same minimums satisfying the "standard relationship" of scattered ordinary and extraordinary waves. It was shown that the normalized scintillation level growth in both non-fully-developed diffraction pattern and in transition zone increasing anisotropy factor. Rising orientation angle scintillation level decreases and splashes arises in fully developed scintillation region.
\end{abstract}

\section{Introduction}

At the present time the features of radio waves propagation in random media have been rather well studied. Many papers and reviews are related to the statistical characteristics of scattered radiation and observations in the ionosphere $[1,2]$. The analysis of the statistical properties of small-amplitude electromagnetic waves that have passed through a plane turbulent plasma slab is very important in many practical applications associated with both natural and laboratory plasmas [3-6].

One of the important research objective is the relationship between scintillation and ionospheric plasmadensity irregularities in the context of space weather and scintillation models. The ionospheric scintillation study comes from its significant impact on satellite radio communications which can degrade the performance of navigation systems and generate errors in received messages. The irregularities distort the original wavefront, giving rise to a randomly phase-modulated wave.

Radio scintillations caused by irregularities in the ionosphere have been extensively observed. In contrast to the large-scale irregularities mostly contributing to phase fluctuations, the small-scale irregularities (from about hundreds of meter to kilometers) mainly affect the amplitude scintillation of radio signals. On propagating to the ground these phase variations cause interference to occur and a diffraction pattern, in both amplitude and phase. Studies of the scintillation, or the temporal and spatial variations, of amplitude and phase of such a signal have yielded much information about the nature of the responsible ionospheric irregularities.

The main task of the scintillation theory is the establishment of the relation between observed statistical characteristics of a wave and properties of an inhomogeneous ionosphere. Three main physical mechanisms cause scintillation: scattering, diffraction and attenuation. These processes should be considered jointly however their relative contribution can change depending on the wavelength, the scale of irregularities, thickness of inhomogeneous slab, distance between the observer to a layer, etc. Generally irregularities are assumed to be "frozen-in" during time of passing by wave and drifting across the direction of propagation with a constant speed. It leads to the drift of the diffraction pattern on the ground, and in the observation point it is perceived as temporary variation of a diffraction pattern.

Usually in scintillation studies, the fluctuations are characterized by a scintillation index whose properties can be related to those of the ionospheric irregularities by application of diffraction theory. Using the power density spectrum of the intensity fluctuations at an observing point on the ground allows study irregularities.

Correlation functions of the phase fluctuations of scattered electromagnetic waves were obtained in the ionospheric $\mathrm{F}$ region normal to the principle plane. Analytical and numerical calculations were carry out for the 
ordinary and extraordinary anisotropic using Gaussian and power-law wavenumber spectra of electron density fluctuations. The scintillation level was estimated for different parameters characterizing elongated irregularities. Scintillation minimums of a temporal spectrum were calculated for different thicknesses of a plasma slab taking into account the movement of rigid irregularities [7]. 2D spectral function of the phase fluctuation was obtained solving differential equation taking into account boundary conditions. Second order statistical moments of the phase fluctuations were calculated considering polarization coefficients of both ordinary and extraordinary waves in the turbulent collision magnetized plasma and the diffraction effects. Analytical and numerical investigations were carried out in the ionospheric $\mathrm{F}$ region for the Gaussian spectral function of electron density fluctuations. Scintillation effects in this region were investigated for the small-scale ionospheric irregularities [8]. The scintillation level is estimated for different anisotropy factors and the orientation angle of elongated irregularities with respect to the static external magnetic field. Scintillation minimums of a temporal spectrum are calculated for different thicknesses of a plasma slab taking into account the movement of rigid irregularities and diffraction effects for scattered ordinary and extraordinary waves in turbulent magnetized plasma [9].

The present paper reports the results of an analysis of the power spectrum of ionospheric scintillation data, which is related to fluctuations in the radio refractive index in the $F$ region. Analytical and numerical calculations of the second order statistical moments of scattered radio waves in the magnetized ionospheric plasma are carried out taking into account diffraction effects. Phase scintillation coefficient and the scintillation level are calculated for spectral function combining both anisotropic Gaussian and power lower spectra. The obtained results are valid for arbitrary correlation function of electron density fluctuations. Numerical calculations are based on the experimental data of the navigation satellites.

\section{Formulation of the Problem}

The electric field in the magnetized turbulent plasma satisfies wave equation:

$$
\left[\nabla_{i} \nabla_{j}-\Delta \delta_{i j}-k_{0}^{2} \varepsilon_{i j}(\mathbf{r})\right] \mathbf{E}_{\mathbf{j}}(\mathbf{r})=0,
$$

components of the second order permittivity tensor are [10]:

$$
\begin{gathered}
\varepsilon_{x x}=1-\frac{\mathrm{v}}{1-u}, \quad \varepsilon_{x y}=-\varepsilon_{y x}=i \frac{\mathrm{v} \sqrt{u} \cos \alpha}{1-u}, \\
\varepsilon_{x z}=-\varepsilon_{z x}=-i \frac{\mathrm{v} \sqrt{u} \sin \alpha}{1-u}, \quad \varepsilon_{y y}=1-\frac{\mathrm{v}\left(1-u \sin ^{2} \alpha\right)}{1-u}, \\
\varepsilon_{y z}=\varepsilon_{z y}=\frac{u \mathrm{v} \sin \alpha \cos \alpha}{1-u}, \quad \varepsilon_{z z}=1-\frac{\mathrm{v}\left(1-u \cos ^{2} \alpha\right)}{1-u}, \\
\mathrm{v}(\mathbf{r})=\frac{\omega_{p}^{2}(\mathbf{r})}{\omega^{2}}, \quad u(\mathbf{r})=\left[\frac{e H_{0}(\mathbf{r})}{m c \omega}\right]^{2}
\end{gathered}
$$

$$
\omega_{p}(\mathbf{r})=\left[\frac{4 \pi N(\mathbf{r}) e^{2}}{m}\right]^{1 / 2},
$$

$\mathrm{v}(\mathbf{r})$ and $u(\mathbf{r})$ are magneto-ionic parameters, $\omega_{p}(\mathbf{r})$ is the plasma frequency, $N(\mathbf{r})$ is the electron density; the static external magnetic field $\mathbf{H}_{\mathbf{0}}$ lying in the $Y Z$ principle plane; $\Delta$ is the Laplacian, $\delta_{i j}$ is the Kronecker symbol, $\alpha$ is the angle between the $Z$-axis (the direction of the wave propagation). At high frequency the effect of ions can be neglected.

Consider a plane wave propagating in the $Z$ direction. Wave field we introduce as [11] $E_{j}(\mathbf{r})=E_{0 j}$ $\exp \left(\varphi_{1}+\varphi_{2}+i k_{\perp} y+i k_{0} z\right) \quad\left(k_{\perp}<<k_{0}\right) \quad, \quad k_{\perp} \quad$ is the wavenumber normal to the principle plane, $k_{0}$ is the wavenumber of an incident wave. Electron density $N(\mathbf{r})=N_{0}+n_{1}(\mathbf{r})$ is a random function of position: $n_{1}(\mathbf{r})$ is assumed to be a homogeneous random field with characteristic spatial scale $l$, the background electron density $N_{0}$ is assumed to be uniform taken as constant; $\mathrm{v}(\mathbf{r})=\mathrm{v}_{0}\left[1+n_{1}(\mathbf{r})\right], \varepsilon_{i j}(\mathbf{r})=\varepsilon_{i j}^{(0)}+\varepsilon_{i j}^{(1)}(\mathbf{r}),\left|\varepsilon_{i j}^{(1)}(\mathbf{r})\right|<<1$. First component represents zero-order approximation; fluctuations of complex phase are of the order $\varphi_{1} \sim \varepsilon_{i j}^{(1)}$. The parameter $\mu=k_{\perp} / k_{0}$ describing diffraction effects is calculated in zero-order approximation.

In the first approximation we have:

$$
\begin{aligned}
& {\left[\nabla_{i} \nabla_{j} \varphi_{1}+\nabla_{i} \varphi_{0} \nabla_{j} \varphi_{1}+\nabla_{i} \varphi_{1} \nabla_{j} \varphi_{0}\right.} \\
& \left.-\delta_{i j}\left(\Delta_{\perp}+2 i k_{\perp} \frac{\partial \varphi_{1}}{\partial y}+2 i k_{0} \frac{\partial \varphi_{1}}{\partial z}\right)-k_{0}^{2} \varepsilon_{i j}^{(0)}\right] E_{0 j}=0
\end{aligned}
$$

where $\Delta_{\perp}=\left(\partial^{2} \varphi_{1} / \partial x^{2}\right)+\left(\partial^{2} \varphi_{1} / \partial y^{2}\right)$ is the transversal Laplasian.

The 2D spectral density function of the phase fluctuation defined by

$$
\varphi_{1}(x, y, z)=\int_{-\infty}^{\infty} d k_{x} \int_{-\infty}^{\infty} d k_{y} \psi\left(k_{x}, k_{y}, z\right) \exp \left(i k_{x} x+i k_{y} y\right)
$$

spatial spectral function in the principle plane (Ycomponent) satisfies differential equation:

$$
\begin{aligned}
& \frac{\partial \psi}{\partial z}+\frac{i d_{1}+d_{2}}{2 k_{0} \mathrm{P}_{j}} \psi(\mathbf{e}, z)=\frac{i k_{0}}{2 \mathrm{P}_{j}}\left[-\varepsilon_{x y}^{(1)}(\mathbf{x}, z)+\mathrm{P}_{j} \varepsilon_{y y}^{(1)}\right. \\
& \left.+\Gamma_{j} \varepsilon_{y z}^{(1)}\right] n_{1}(\mathbf{e}, z)
\end{aligned}
$$

where: $d_{1}=k_{0} k_{y} \Gamma_{j}-k_{x}^{2} \mathrm{P}_{j}, d_{2}=k_{x}\left(k_{y}+k_{\perp}\right)$.

Polarization coefficients are defined as [10]:

$$
\mathrm{P}_{j}=\frac{2 \sqrt{u}(1-\mathrm{v}) \cos \alpha}{u \sin ^{2} \alpha \pm \sqrt{u^{2} \sin ^{4} \alpha+4 u(1-\mathrm{v})^{2} \cos ^{2} \alpha}},
$$




$$
\Gamma_{j}=-\frac{\mathrm{v} \sqrt{u} \sin \alpha+\mathrm{P}_{j} u \mathrm{v} \sin \alpha \cos \alpha}{1-u-\mathrm{v}+u \mathrm{v} \cos ^{2} \alpha},
$$

upper sign $(j=1)$ before square root corresponds to the ordinary wave, lower sign to the extraordinary wave $(j=2)$. External magnetic field changes electromagnetic properties of plasma make it magnetized (birefringence, gyrotropic and anisotropic) medium. Gyrotropy of plasma is revealed in elliptic polarization of normal waves; anisotropy is appeared in the direction of propagation depending of their characteristics (polarization, refractive index and absorption).

Solving Equation (4) and using the boundary condition $\psi(\mathfrak{x}, z=0)=0$ we calculate statistical characteristics of scattered electromagnetic waves. The variance of the phase fluctuation is:

$$
<\varphi_{1}^{2}(\mathbf{r})>=-\frac{\pi}{2} \frac{k_{0} L}{\mathrm{P}_{j}^{2}} \int_{-\infty}^{\infty} d k_{x} \int_{-\infty}^{\infty} d k_{y} Q_{1}\left(k_{x}, k_{y}\right),
$$

where: $\quad Q_{1}\left(k_{x}, k_{y}\right)=V_{x y, x y}+\mathrm{P}_{j}^{2} V_{y y, y y}+\Gamma_{j}^{2} V_{y z, y z}-2\left(\mathrm{P}_{j}\right.$ $\left.V_{x y, y y}+\Gamma_{j} V_{x y, y z}+\mathrm{P}_{j} \Gamma_{j} V_{y y, y z}\right)$ contains arbitrary 3D spectral correlation function of electron density fluctuation, $V_{\alpha \beta, \gamma \delta}\left(\mathfrak{e}, i G_{3}-G_{4}\right), \mathfrak{x}=\left\{k_{x}, k_{y}\right\}, L$ is the distance travelling by electromagnetic wave in the ionospheric plasma, the angular brackets indicate the statistical average, $G_{3}=k_{x} k_{\perp} / 2 k_{0} \mathrm{P}_{j}, G_{4}=k_{0} k_{y} \Gamma_{j} /\left(2 k_{0} \mathrm{P}_{j}\right)$.

Correlation function of the phase fluctuations is:

$$
\begin{aligned}
& W_{\varphi}(\boldsymbol{\rho})>\equiv<\varphi_{1}(\mathbf{r}) \varphi_{1}^{*}(\mathbf{r}+\boldsymbol{\rho})>=\frac{\pi}{2} \frac{k_{0}^{2} L}{\mathrm{P}_{j}^{2}} \int_{-\infty}^{\infty} d k_{x} \int_{-\infty}^{\infty} d k_{y} \\
& Q_{2}\left(k_{x}, k_{y}\right) \exp \left(-i k_{x} \rho_{x}-i k_{y} \rho_{y}\right),
\end{aligned}
$$

where: $Q_{2}\left(k_{x}, k_{y}\right)$ function contains $V_{\alpha \beta, \gamma \delta}\left(\mathbf{x}, G_{5}\right)$, $G_{5}=k_{0} k_{x}\left(\mathrm{P}_{j} k_{x}+i k_{\perp}\right) / 2 \mathrm{P}_{j} k_{0}^{2}, \rho_{x}$ and $\rho_{y}$ are distances between observation points spaced apart in the principle and perpendicular planes, respectively. The double integral in the wave number space depends only on the shape of the fluctuation spectrum but not on the strength of the fluctuations. Phase fluctuations at different observation points are not independent and they correlate. The asterisk indicates the complex conjugate. The spatial autocorrelation function of the diffraction pattern could be measured with a suitable 2D array of sensors.

The scintillation level $S_{4}$ for weak scattering is determined by the 2D phase spectrum describing diffraction pattern on the ground is [12]:

$$
S_{4}^{2}=2 \int_{-\infty}^{\infty} d k_{x} \int_{-\infty}^{\infty} d k_{y} W_{\varphi}\left(k_{x}, k_{y}, L\right) \sin ^{2}\left(\frac{k_{x}^{2}+k_{y}^{2}}{k_{f}^{2}}\right),
$$

where: $k_{f}=(4 \pi / \lambda z)^{1 / 2}$ is the Fresnel wavenumber. The double integral in the wave number space not depends on the intensity fluctuations and depends on the shape of the fluctuation spectrum. The sinusoidal term is responsible for oscillations in the scintillation spectrum. The value of the scintillation index depends on geometry, frequency and the ionization irregularity structure. Phase scintillations are usually observed as a phase difference between the antennas of an interferometer system.

If rigid irregularities are moving past the radio ray trajectory along the $X$ direction with apparent velocity $V_{x}$, scintillation level $S_{4}$ is computed from the power spectrum [13]; the relationship between the power spectrum of the intensity fluctuations $P_{S}(v)$ and the power spectrum of the phase fluctuations $P_{\varphi}(v)$ for one dimensional (the irregularities are elongated perpendicular to their direction of motion) weak scattering screen is given by [14]:

$$
\begin{gathered}
S_{4}^{2}=\int_{0}^{\infty} d v P_{S}(v), P_{\varphi}(v)=\frac{2 \pi}{k_{0} V_{x}} \int_{0}^{\infty} d y \tilde{W}_{\varphi}\left(x=\frac{v}{v_{0}}, y\right), \\
P_{S}(v)=4 P_{\varphi}(v) \sin ^{2}\left(\frac{v}{v_{f}}\right)^{2}
\end{gathered}
$$

where: $v_{0}=k_{0} V_{x} / 2 \pi$, the Fresnel frequency, $v_{f}=V_{x}$ $(\pi \lambda z)^{-1 / 2}$ is directly proportional to the drift velocity $V_{x}$ transverse to the radio path and inversely proportional to the Fresnel radius $F=(\lambda z)^{1 / 2}, \lambda$ is the radio wavelength and $Z$ is the mean distance between the observer and the irregularities, $v$ is the scintillation frequency. The sinusoidal term is responsible for oscillations in the scintillation spectrum.

\section{Numerical Calculations}

The incident electromagnetic wave has the frequency of $3 \mathrm{MHz}\left(k_{0}=6.28 \cdot 10^{-2} \mathrm{~m}^{-1}\right)$ propagates along the $Z$-axis. Plasma parameters at the altitude of $300 \mathrm{~km}$ are: $u_{0}=0.22$, $\mathrm{v}_{0}=0.28$. Numerical calculations are carried out for the angle $\alpha=20^{\circ}$ between the incident wave propagation and the external magnetic field. The first Fresnel radius and the Fresnel wavenumber are equal to $5.5 \mathrm{~km}$ and $0.64 \mathrm{~km}^{-1}$, respectively.

An RH-560 rocket flight observations from Sriharikota rocket range (SHAR), India $\left(14^{0} \mathrm{~N}, 80^{\circ} \mathrm{E}\right.$, dip latitude $5.5^{0} \mathrm{~N}$; apogee was $348 \mathrm{~km}$ ) show [15] that the intermediate range irregularities $(100 \mathrm{~m}-2 \mathrm{~km})$ were observed in abundance in altitude regions $220-250 \mathrm{~km}$ and $290-320 \mathrm{~km}$. Irregularities of a range of scale sizes starting from a few hundred meters to a few ten of kilometers are observed in these patches. Analyses of electron density data show that the spectra of transitional scales are quite steep having an average spectral index of 4.32 on the altitude about $300 \mathrm{~km}$. During this flight the gradient drift instability was responsible for the generation of irregularities in the intermediate scales. Studying the equatorial spread $\mathrm{F}$ irregularities using $\mathrm{RH}-560$ rocket 
instrumented with Langmuir probes launched from SHAR, India, it was established [16] that the relationship between the spectral index, $p$ and the mean integrated spectral power (in $20 \mathrm{~m}$ to $200 \mathrm{~m}$ scale size range) could be represented by a Gaussian function.

The general shape of the power spectrum for the irregularities is the power-law type with the power index between 2 and 4. For a typical ionosphere the irregularity spectrum is a power law with exponent $p \sim 3$ showed that, for this spectrum of irregularities, $\left\langle\varphi_{1}^{2}>\sim 10^{3}\right.$ radians $^{2}$ produces a scintillation index approximately equal 1 , and $<\varphi_{1}^{2}>\sim 10^{-1}$ radians $^{2}$ produces a weak scintillation index approximately equal to 0.1 [17].

The reciprocal oblique sounding method based on the reception of backscattered signals has been used [18] for the artificial irregularity diagnostics on different length paths. The experiments were carried out using "Sura" heating facility in the frequency range of 4.7-9 MHz. Wideband antennae were spaced at distances from 75 to $250 \mathrm{~m}$ apart. The magnitudes of the drift velocity measured were between 65 and $270 \mathrm{~meter} / \mathrm{sec}$; the typical velocities of ionospheric $V_{d r}=60-100 \mathrm{~meter} / \mathrm{sec}$. Spectral index was equal to $p=1.4-4.8$ in different heating sessions. Relative variation of electron density fluctuations was of the order $(1-5) \cdot 10^{-3}$. Observations (Tbilisi, $41^{0} 43 \mathrm{~N}$ ) of drift small-scale irregularities in the ionospheric F-region show [19] that they have elliptic form, the ratio of axes basically varies from 1 to 3. Anisotropy axis is mainly oriented along the geomagnetic field of lines. Drift of small-scale irregularities mainly has S-W direction. The most probable values of drift velocity is in the range of 40-100 meter/sec.

Spectral function of electron density irregularities combining anisotropic Gaussian and power-law spectra is:

$$
\begin{aligned}
& V_{n}(\mathbf{k})=\frac{\sigma_{n}^{2}}{8 \pi^{5 / 2}} \frac{A_{p} l_{\|}^{3}}{\chi^{2}\left[1+l_{\perp}\left(k_{x}^{2}+k_{y}^{2}\right)+l_{\|} k_{z}^{2}\right]^{p / 2}} \\
& \exp \left[\left(-\frac{k_{x}^{2} l_{\perp}^{2}}{4}-p_{1} \frac{k_{y}^{2} l_{\|}^{2}}{4}-p_{2} \frac{k_{z}^{2} l_{\|}^{2}}{4}+p_{3} k_{y} k_{z} l_{\|}^{2}\right)\right],
\end{aligned}
$$

where: $p_{1}=\left(\sin ^{2} \gamma_{0}+\chi^{2} \cos ^{2} \gamma_{0}\right)^{-1}\left[1+\left(\chi^{2}-1\right)^{2} \sin ^{2} \gamma_{0}\right.$

$\left.\cdot \cos ^{2} \gamma_{0} / \chi^{2}\right], p_{2}=\left(\sin ^{2} \gamma_{0}+\chi^{2} \cos ^{2} \gamma_{0}\right) / \chi^{2}$,

$p_{3}=\left(\chi^{2}-1\right) \sin \gamma_{0} \cos \gamma_{0} / 2 \chi^{2}, \quad A_{p}=\Gamma\left(\frac{p}{2}\right) \Gamma\left(\frac{5-p}{2}\right)$

$\cdot \sin \left[\frac{(p-3) \pi}{2}\right], \Gamma(x)$ is the gamma function, $p$ is the power index; $k_{x}, k_{y}$ and $k_{z}$ are the wave vector $\mathbf{k}$ components perpendicular $\left(k_{x}, k_{y}\right)$ and parallel $\left(k_{\|}\right)$to the propagation direction, $\chi=l_{\|} / l_{\perp}$ is the anisotropy coefficient - the ratio of longitudinal and transverse characteristic linear sizes of plasma irregularities, $\gamma_{0}$ is the orientation angle of elongated irregularities with respect to the lines of force of geomagnetic. The shape of electron density irregularities has a spheroidal form. The temporal power spectrum of the radio-wave scintillation is related to the in situ spatial spectrum of the scintillation producing irregularities in ionospheric electron density. Scintillation spectra are in agreement with $3 \mathrm{D}$ power-law irregularity spectrum with an spectral index 4 [14] and with the in situ measurements of the one-dimensional irregularity spectrum derived from rockets and satellites.

Substituting (9) into equations (4) and (5) at $p=4$ the variance and correlation function of the phase fluctuations have the following forms:

$$
\begin{aligned}
& <\varphi_{1}^{2}(\mathbf{r})>=\sigma_{n}^{2} \frac{\xi^{3} k_{0} L}{16 \sqrt{\pi}} \frac{A_{0} A_{p}}{\mathrm{P}_{j}^{2} \chi^{2}} \int_{-\infty}^{\infty} d x \int_{-\infty}^{\infty} d y \exp (-i g x y) \\
& \frac{\left(h_{3}^{2}-h_{2}^{2}\right)-2 i h_{2} h_{3}}{\left(h_{3}^{2}-h_{2}^{2}\right)^{2}+4 h_{2}^{2} h_{3}^{2}} \exp \left[-\frac{\xi^{2}}{4}\left(a x^{2}+b y^{2}\right)\right] \text {, } \\
& W_{\varphi}\left(\eta_{x}, \eta_{y}, L\right)=\sigma_{n}^{2} \frac{\xi^{3} k_{0} L}{16 \sqrt{\pi}} \frac{A_{0} A_{p}}{\mathrm{P}_{j}^{2} \chi^{2}} \int_{-\infty}^{\infty} d x \int_{-\infty}^{\infty} d y \\
& \frac{\left(h_{3}^{2}-h_{2}^{2}\right)-2 i h_{2} h_{3}}{\left(h_{3}^{2}-h_{2}^{2}\right)^{2}+4 h_{2}^{2} h_{3}^{2}} \cdot \exp \left(-\frac{\xi^{2}}{4} p_{1} y^{2}-i \eta_{y} y\right) \\
& \exp \left\{-\frac{\xi^{2}}{4}\left[\frac{p_{2}}{4} x^{4}+\left(\frac{1}{\chi^{2}}-\frac{p_{2} \mu^{2}}{4 \mathrm{P}_{j}^{2}}+2 p_{3} y\right) x^{2}\right]\right\} \\
& \exp \left\{-i \frac{\xi^{2}}{4}\left[\frac{\mu}{2 \mathrm{P}_{j}}\left(x^{3}+4 p_{3} x y\right]-i \eta_{x} x\right\}\right.
\end{aligned}
$$

where: $a=\frac{1}{\chi^{2}}-\frac{p_{2} \mu^{2}}{4 \mathrm{P}_{j}^{2}}, b=p_{1}+\frac{p_{2}}{4} \frac{\Gamma_{j}^{2}}{\mathrm{P}_{j}^{2}}-2 p_{3} \frac{\Gamma_{j}}{\mathrm{P}_{j}}$,

$$
g=\frac{\xi^{2}}{2} \frac{\mu}{\mathrm{P}_{j}}\left(p_{3}-\frac{p_{2}}{4} \frac{\mathrm{P}_{j}}{\Gamma_{j}}\right), \quad \eta_{x}=k_{0} \rho_{x}, \eta_{y}=k_{0} \rho_{y},
$$$$
h_{1}=\frac{\chi^{2}}{4} x^{4}+\left(1-\frac{\chi^{2} \mu^{2}}{4 \mathrm{P}_{j}^{2}}\right) x^{2}, \quad h_{2}=\frac{\mu \chi^{2}}{2 \mathrm{P}_{j}}
$$$$
h_{3}=1+\frac{\xi^{2}}{\chi^{2}}\left(h_{1}+y^{2}\right), \quad A_{0}=\frac{\mathrm{v}_{0}^{2}}{\left(1-u_{0}\right)^{2}}\left[u_{0} \cos \alpha+\mathrm{P}_{j}^{2}\right.
$$$$
\left.\left(1-u_{0} \sin ^{2} \alpha\right)^{2}+\Gamma_{j}^{2} u_{u}^{2} \sin ^{2} \alpha \cos ^{2} \alpha\right]-2 \frac{\mathrm{v}_{0}^{2} \sqrt{u_{0}} \cos \alpha}{\left(1-u_{0}\right)^{2}}
$$

$\left[\mathrm{P}_{j}\left(1-u_{0} \sin ^{2} \alpha\right)+\Gamma_{j} u_{0} \sin \alpha \cos \alpha+\mathrm{P}_{j} \Gamma_{j} \sqrt{u_{0}}\right.$

$$
\text { . } \left.\left(1-u_{0} \sin ^{2} \alpha\right)\right] \text {. The double integral in equation }
$$
and (11) depends only on the shape of the spectrum and is independent of the variance of density fluctuations. The layer of strong irregularities is often $100-500 \mathrm{~km}$ thick ranging from $200-700 \mathrm{~km}$ in altitude [20]. 
If $p=4$ and $\xi=k_{0} l_{\|}>1 \quad\left(l_{\|}\right.$is characteristic linear scale of ionospheric plasma irregularities along the static external magnetic field) and the altitude of the background density variations is much greater than both $\lambda$ and $l_{\|}$ (these assumptions are well satisfied for the ionosphere scintillation case) equations (10) and (11) yield:

$$
\begin{gathered}
<\varphi_{1}^{2}>=\sigma_{n}^{2} \frac{\sqrt{\pi}}{4} \frac{A_{0} \xi k_{0} L}{\chi^{2} \sqrt{b} \mathrm{P}_{j}^{2}}\left(\frac{1}{\chi^{2}}-\frac{p_{2} \mu^{2}}{4 \mathrm{P}_{j}^{2}}\right), \\
W_{\varphi}\left(\eta_{y}, L\right)=\sigma_{n}^{2} \frac{\xi^{2} k_{0} L}{8} \frac{A_{0}}{\mathrm{P}_{j}^{2} \chi^{2}} \int_{-\infty}^{\infty} d y\left(1+\frac{\xi^{2}}{\chi^{2}} y^{2}\right)^{-2} \\
\left(\frac{1}{\chi^{2}}-\frac{p_{2} \mu^{2}}{4 \mathrm{P}^{2}}+2 p_{3} y\right)^{-1 / 2} \exp \left(-\frac{\xi^{2}}{4} p_{1} y^{2}-i \eta_{y} y\right) .
\end{gathered}
$$

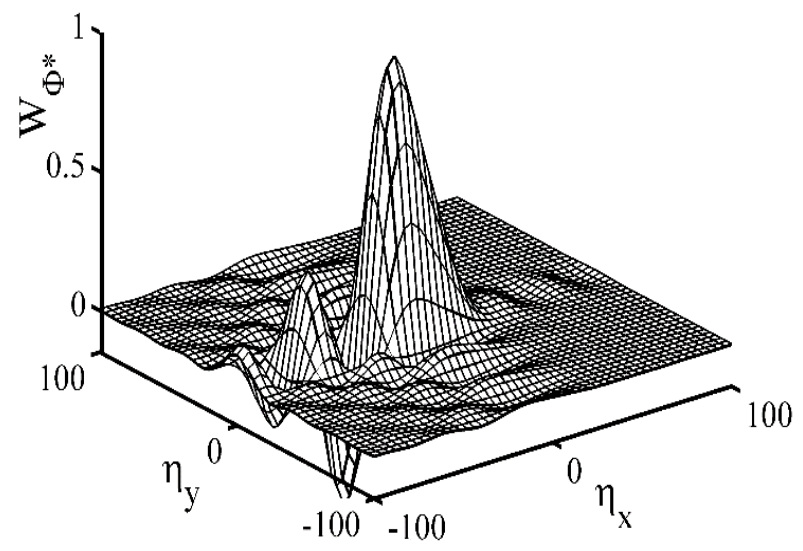

Figure 1: Normalized correlation function of the phase fluctuations for the anisotropic Gaussian spectral function characterizing random variations of electron density versus distances between observation points.

At $\mu=0, H_{0}=0, A_{0}=\mathrm{v}_{0}^{2}$, we obtain the well-known result $\left\langle\varphi_{1}^{2}\right\rangle=\sigma_{n}^{2} \sqrt{\pi} \mathrm{v}_{0}^{2} \xi k_{0} L / 4$ [3].

Figures 1 and 2 represents normalized correlation function of the phase fluctuations $W_{\varphi^{*}}=W_{\varphi}\left(\eta_{x}, \eta_{y}, L\right) / W_{\varphi}(0,0, L)$ for elongated $\left(\gamma_{0}=0^{0}\right)$ plasma irregularities: $\xi=300,\left(l_{\|}=4.5 \mathrm{~km}\right), \chi=30$. Oscillations arise by Gaussian spectrum; power-law spectrum flattens these oscillations.

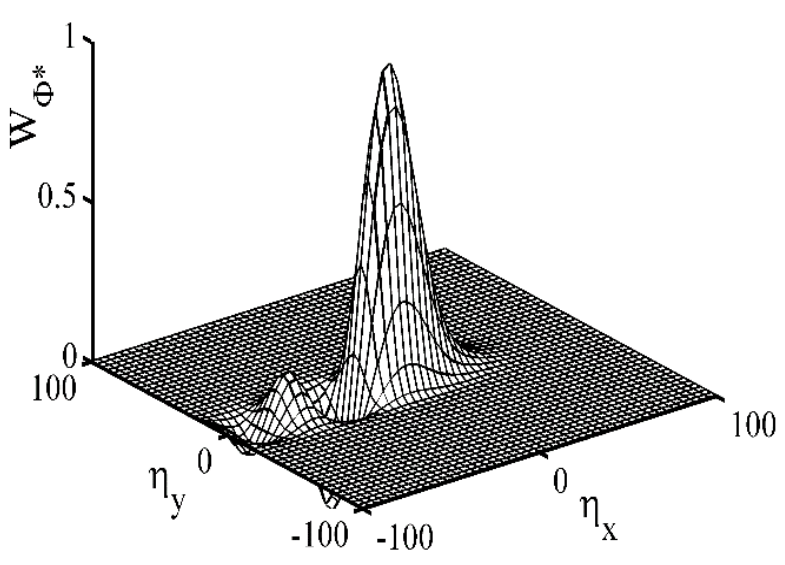

Figure 1: The same as in Figure 1 for spectral function (13).

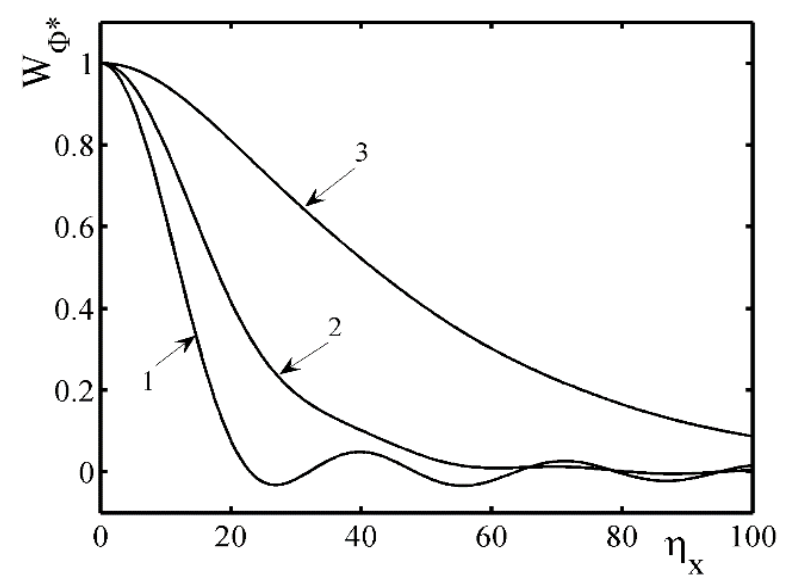

Figure 3: Normalized correlation function of the phase fluctuations versus $\eta_{x}$ for the spectrum (9).

Figures 3 and 4 depicts the dependence of the normalized correlation function of the phase fluctuations versus $\eta_{x}$ (observation points spaced apart normal to the principle plane) for the ordinary waves if small-scale irregularities strongly elongated along the static external magnetic field $\left(\gamma_{0}=0^{0}\right), \mu=0.06, \chi=2$ at: $\xi=10$ $\left(l_{\|}=160 \mathrm{~m}\right)$, curve $1, \xi=20\left(l_{\|}=320 \mathrm{~m}\right)$ curve $2, \xi=50$ $\left(l_{\|}=800 \mathrm{~m}\right)$ curve 3 . Increasing characteristic linear scales of irregularities oscillations become smooth for both spectra. For small parameter $\xi$ the behavior of the ordinary and extraordinary wave approximately are the same. 
Figures 5 and 6 illustrates ordinary and extraordinary waves at: $\gamma_{0}=0^{0}, \mu=0.06, \chi=10$ for irregularities having linear scales $1.5 \mathrm{~km}$ and $3 \mathrm{~km}$, respectively. At

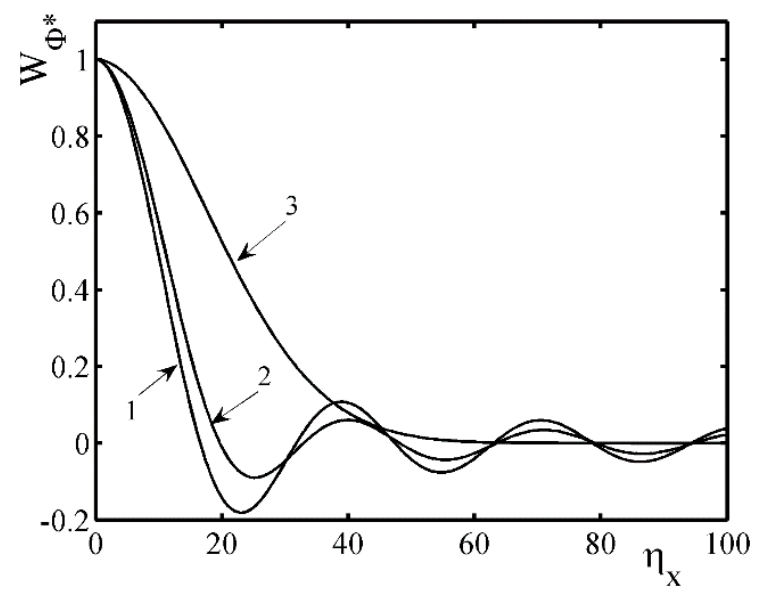

Figure 4: Normalized correlation function of the phase fluctuations versus $\eta_{x}$ for the Gaussian spectrum.

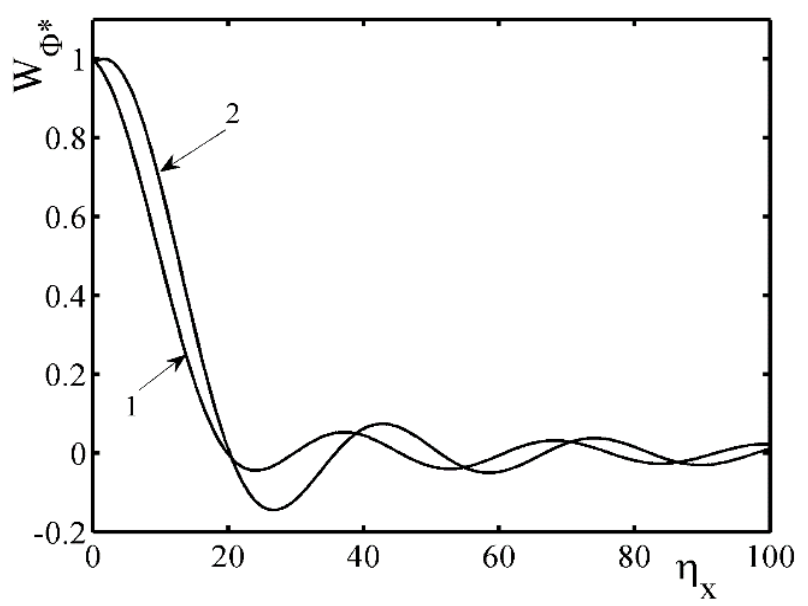

Figure 5: Ordinary (curve 1) and extraordinary (curve 2) waves at $\xi=100$.

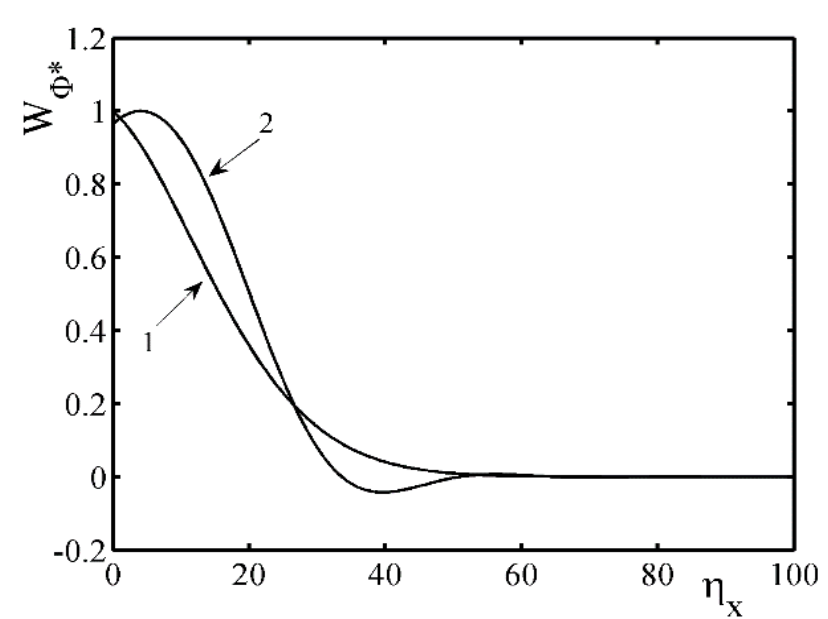

Figure 6: The same as on figure 5 at $\xi=200$. $\xi=100$ both wave oscillates, while if $\xi=200$ when distance between observation points is in the interval $0 \leq \eta_{x}<25$ extraordinary wave dominates, at $25 \leq \eta_{x}<60$ vice versa; starting $\eta_{x} \geq 60$ the behavior of both wave is the same.

Anisotropy factor $\chi$ and characteristic linear scale $l_{\|}$have a substantial influences on the ordinary and extraordinary waves. Variances of the phase fluctuation are calculated at $\gamma_{0}=0^{0}$ both for the anisotropic Gaussian spectrum

$$
<\varphi_{1}^{2}>=\sqrt{\pi} \sigma_{n}^{2} \xi k_{0} L \frac{A_{0}}{\mathrm{P}_{j}^{2} \chi^{2}} a^{-1 / 2}\left(b-\frac{4 g^{2}}{a \xi^{4}}\right)^{-1 / 2}
$$

and equation (12) for the spectral function (9). Applying (14) for small-scale irregularities at $\xi=20\left(l_{\|}=300 \mathrm{~m}\right)$ and $\chi=10\left(l_{\perp}=30 \mathrm{~m}\right)$ we have $\left\langle\varphi_{1}^{2}\right\rangle=0.055$; for large-scale irregularities using (13), at $\xi=300\left(l_{\|}=4.8\right.$ $\mathrm{km})$ and $\chi=5 \quad\left(l_{\perp}=1 \mathrm{~km}\right)$ we obtain $\left\langle\varphi_{1}^{2}\right\rangle=0.11$.

Figures 7 and 8 depicts the phase scintillation index $S_{4}$ versus anisotropy factor $\chi$. Plasma irregularities strongly elongated along the external magnetic field $\left(\gamma_{0}=0^{\circ}\right)$ and $L=400 \mathrm{~m}$ (Figure 7). Increasing characteristic spatial scale of irregularities from $l_{\|}=1.6 \mathrm{~km}$ up to $l_{\|}=6.4 \mathrm{~km}$ scintillation index increases approximately twice. Curve 1 corresponds $\xi=100, S_{4 \max }=0.46$ at $\chi=3$; curve $2-\xi=$ $200, S_{4 \max }=0.65$ at $\chi=7$; curve $3-\xi=400, S_{4 \max }=$ 0.92 at $\chi=13$. Reaching maximums the curves tends to the saturation. This is in agreement with [21]. For fixed $\xi=100\left(l_{\|}=1.5 \mathrm{~km}\right)$ and $L=300 \mathrm{~m}$ (Figure 8), varying orientation angle of elongated large-scale plasma irregularities with respect to the lines of force of the geomagnetic field splashes arises in the scintillation index. Inflection point of these curves is at $\chi=2.4$.

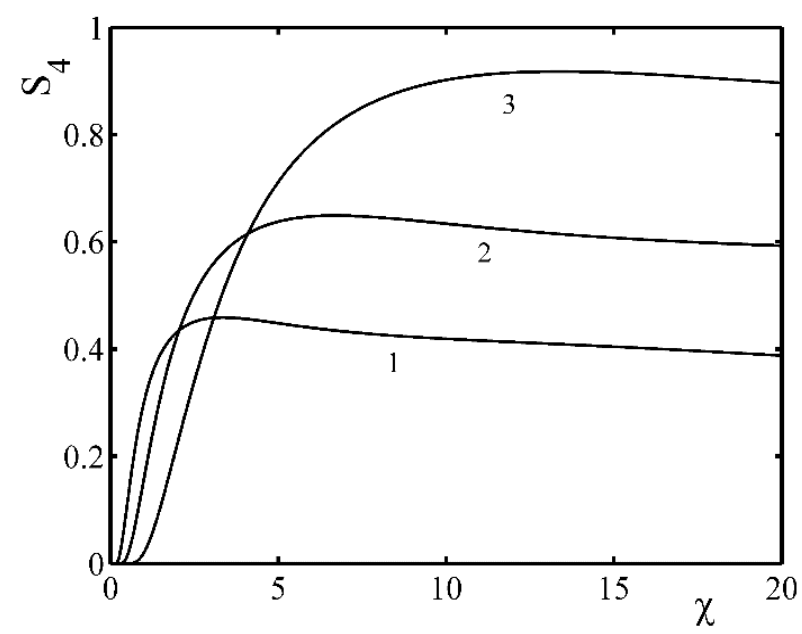

Figure 7: Scintillation index versus anisotropy coefficient $\chi$ for different parameter $\xi$ at $k_{0} L=25$. 


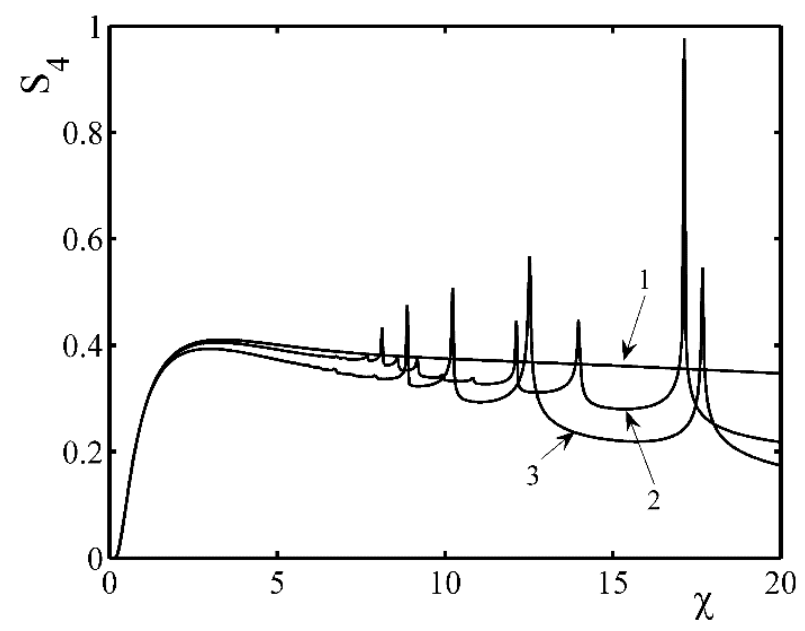

Figure 8: Scintillation index versus anisotropy coefficient $\chi$ for different parameter $\xi$ at $k_{0} L=20$.

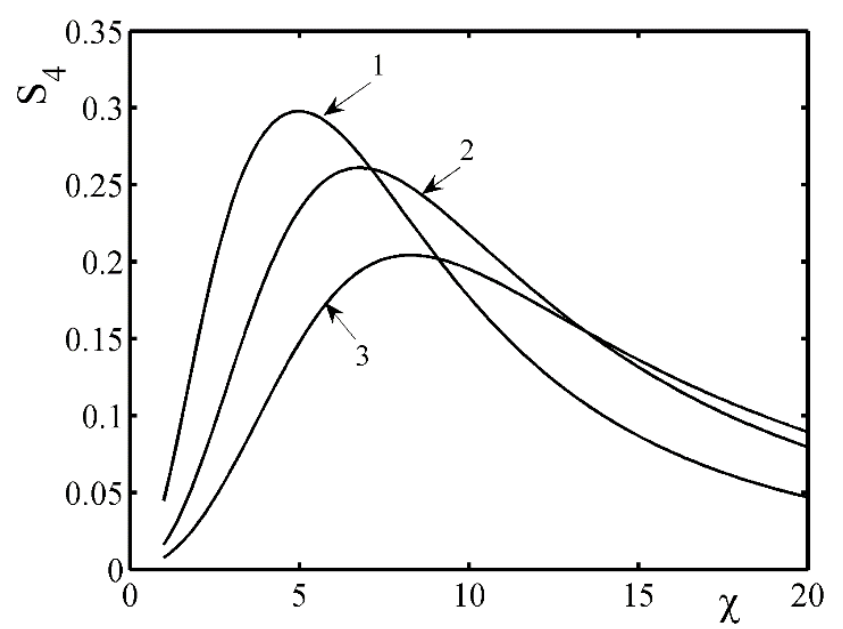

Figure 9: Scintillation index versus anisotropy coefficient $\chi$ for different parameter $\xi$.

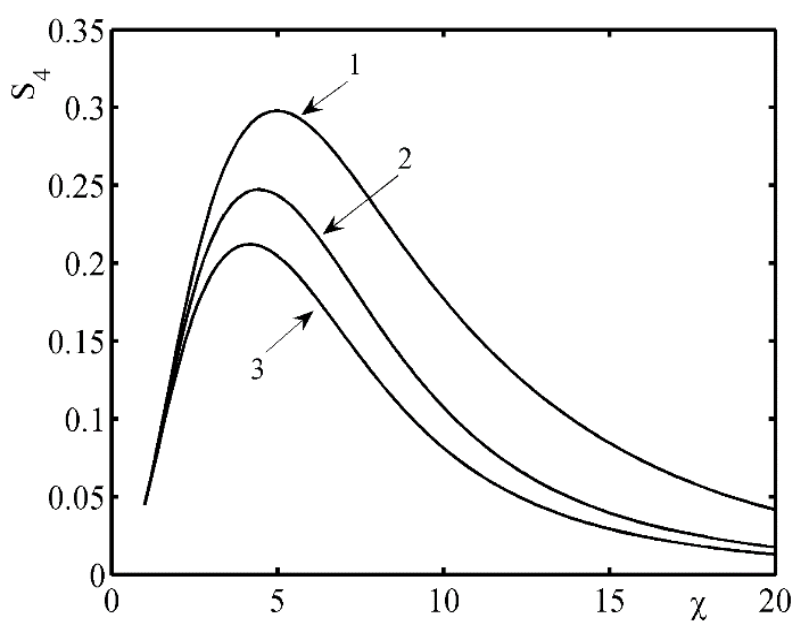

Figure 10: Scintillation index versus anisotropy coefficient $z$ for different orientation angle $\gamma_{0}$.
Curve $1 \quad\left(\gamma_{0}=0^{0}\right)$ - not splashes, curve $2\left(\gamma_{0}=10^{0}\right)$ splashes arises at $\chi=11,12,14,17$; curve $3\left(\gamma_{0}=20^{\circ}\right)$ splashes are revealed at $\chi=9,10,12,18$. Scattering by irregularities whose scale is of the order of $F$ or less. Known as diffractive scattering, involves scattering through small angles of about $\lambda /(2 \pi F)$.

Figures 9 and 10 show the phase scintillation index $S_{4}$ as a function of anisotropy factor of strongly elongated $\left(\gamma_{0}=0^{0}\right)$ small-scale ionospheric plasma irregularities using the temporal power spectrum (8) and spectral function (9), taking into account "frozen-in" assumption for the irregularities. The Gaussian hump is the general behavior for the scintillation spectrum on both figures. The following parameters are chosen for computations: for the frequency of an incident wave $3 \mathrm{MHz}$ the Fresnel radius $F=4 \mathrm{~km}$, the Fresnel frequency is $v_{f}=0.01 \mathrm{~s}^{-1}, \alpha=20^{0}$, $\mu=0.06$. Scintillation index increases at small anisotropy parameter $\chi$ reaching maximums and then decreases in proportion to the linear scale of irregularities. Curve 1 corresponds: $\xi=15$ (characteristic spatial scale is $\left(l_{\|}=240 \mathrm{~m}\right), S_{4 \max }=0.3$ is at $\chi=5$; curve $2-\xi=30$ $\left(l_{\|}=480 \mathrm{~m}\right), S_{4 \max }=0.26$ is at $\chi=7$; curve $3-\xi=50$ $\left(l_{\|}=800 \mathrm{~m}\right) . S_{4 \max }=0.2$ is at $\chi=8$. Figure 8 depicts phase scintillation index for $l_{\|}=240 \mathrm{~m}$. Curve 1: $\gamma_{0}=0^{0}$, at $\chi=5, S_{4 \max }=0.3$; curve $2: \gamma_{0}=15^{0}$, at $\chi=4.4$, $S_{4 \max }=0.25$; curve $3: \gamma_{0}=30^{\circ}$, at $\chi=4.2, S_{4}=0.21$. Increasing slop angle $\gamma_{0}$ of elongated irregularities maximum of the scintillation index $S_{4}$ shifts to the left. Maximum $S_{4 \max }$ is in the interval $0.2-0.3$ indicating conditions of weak scatter of the radio wave by the ionospheric irregularities. The relationship between the spectral index and the intensity of the scintillation characterizing by the $S_{4}$ index show that all the observed $S_{4}$ values are less than 0.55 , moderate scintillation intensity, within the weak-scatter regime. The obtained results can be applied in determining the anisotropy parameters of the ionospheric irregularities from observed statistical parameters of the phase fluctuations.

Observation of the scintillation of radio waves is connected with the investigation of the diffraction pattern on the ground level. Structure of the diffraction pattern substantially depends as on the phase fluctuations in the inhomogeneous slab as well as on the ratio of the linear scale of plasma irregularities and the Fresnel radius. Ionospheric scintillation depends on the 2D spectral correlation function of electron concentration fluctuations. The two asymptotic simplifications $\Upsilon<<1$ is associated with a significant filtering, non-fully developed diffraction pattern, whereas $\Upsilon>>1$ is associated with a fully 
developed scintillation. Shaded area corresponds to a transition region between these two regions.

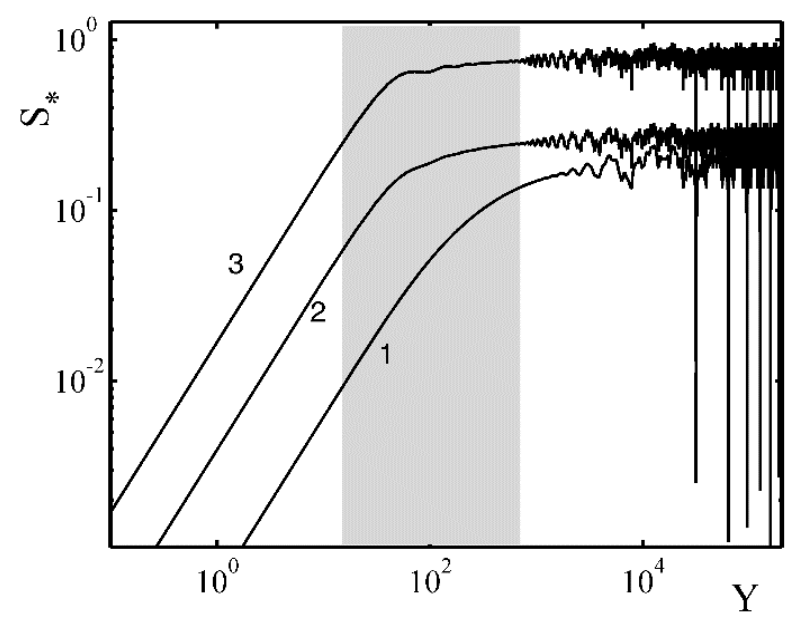

Figure 11. Normalized scintillation level versus parameter $\Upsilon$ for different parameter $\chi$.

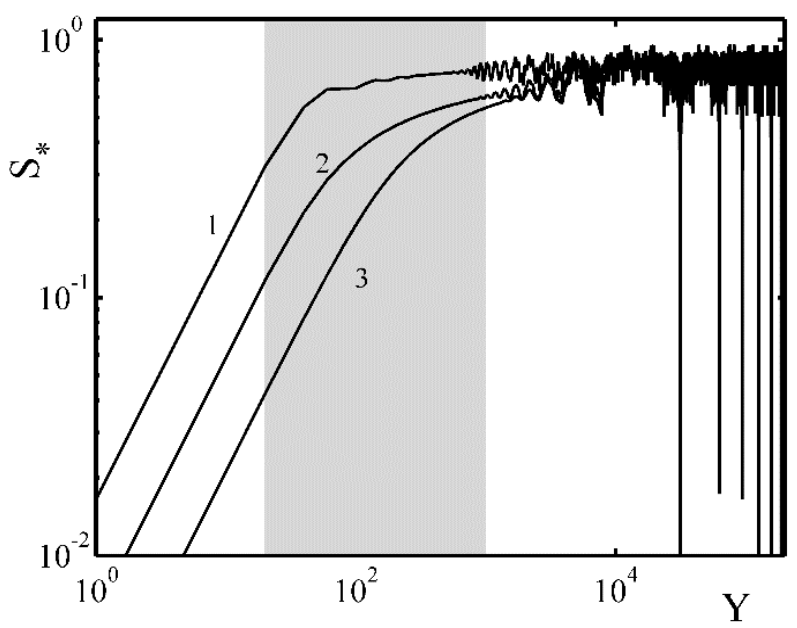

Figure 12: Normalized scintillation level versus parameter $\Upsilon$ for different orientation angle $\gamma_{0}$.

Figures 11 depicts the log-log plots of the normalized scintillation level $S_{*}=S_{4} /\left\langle\varphi_{1}^{2}\right\rangle^{1 / 2}$ versus parameter $\Upsilon=2\left(k_{0} / k_{f}\right)^{2}, \quad \mu=5 \cdot 10^{-3}, \gamma_{0}=0^{0}, \alpha=20^{0} \quad$ and $\xi=100 \quad\left(l_{\|}=1.6 \mathrm{~km}\right)$. Increasing anisotropy factor scintillation index increases in both non-fully developed diffraction pattern $(\Upsilon<<1)$ and a transition region (shaded area). No oscillations in a transition region. Oscillations arises at $\Upsilon=1940\left(k_{f}=2 \mathrm{~km}^{-1}\right)$ for $\chi=5$; at $\Upsilon=920$ $\left(k_{f}=3 \mathrm{~km}^{-1}\right)$ for $\chi=15$, at $\Upsilon=500\left(k_{f}=4 \mathrm{~km}^{-1}\right)$ for $\chi=30$. Splashes are revealed in the region $\Upsilon>>1$. Particularly at $\Upsilon=3.14 \cdot 10^{4} \quad\left(k_{f}=0.5 \quad \mathrm{~km}^{-1}\right)$, $\Upsilon=6.28 \cdot 10^{4}\left(k_{f}=0.35 \mathrm{~km}^{-1}\right), \Upsilon=9.42 \cdot 10^{4},\left(k_{f}=0.29\right.$ $\left.\mathrm{km}^{-1}\right)$ and $\Upsilon=12.56 \cdot 10^{4}\left(k_{f}=0.25 \mathrm{~km}^{-1}\right)$. Hence parameter $\Upsilon$ satisfies the proportion: $1: 2: 3: 4, \ldots$ Figure 12 represents the log-log plots of the of the normalized scintillation level versus parameter $\Upsilon$ at $\chi=30$.

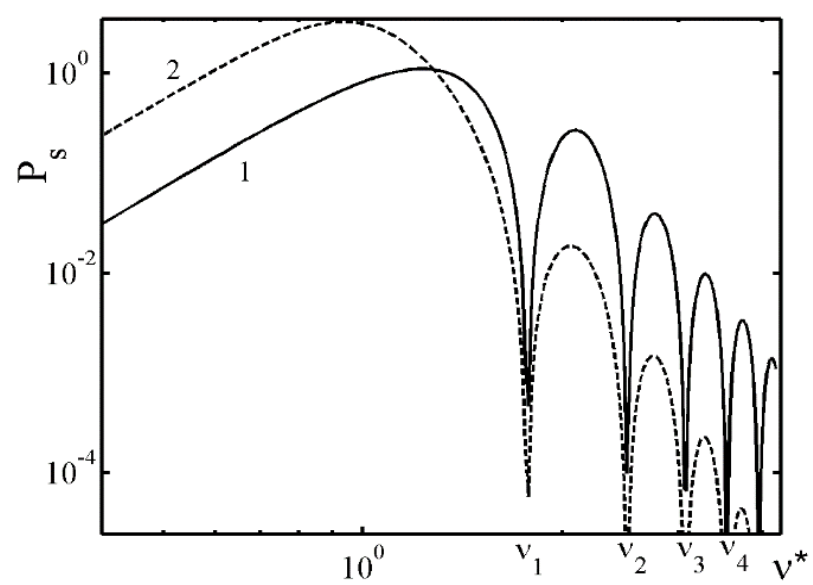

Figure 13: Power spectrum of the intensity fluctuations as log-log plots of the frequency parameter $v_{*}=v / v_{f}$. Irregularities are moving along the $X$-axis.

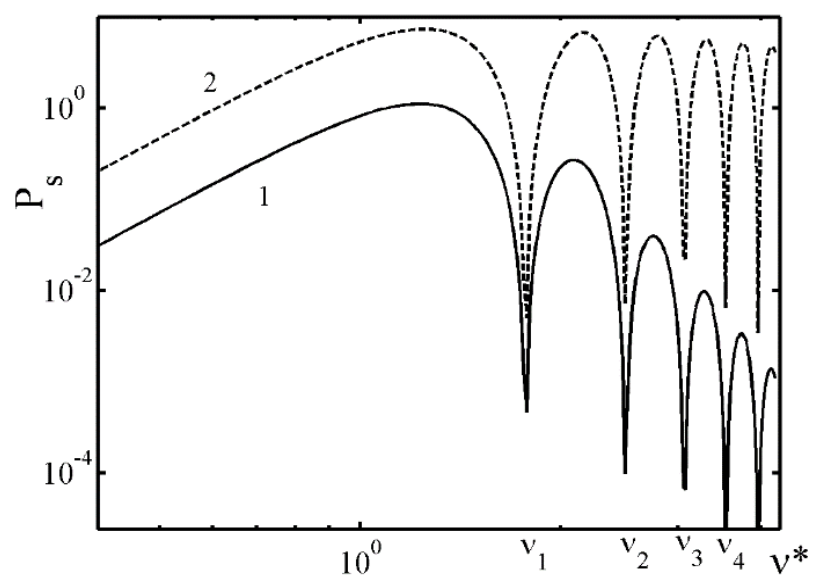

Figure 14: Power spectrum of the intensity fluctuations as $\log -\log$ plots of the frequency parameter $v_{*}=v / v_{f}$. Irregularities are moving along the $X$-axis (solid line); along the $Y$-axis (dashed line).

Varying slop angle in the interval $0^{0} \leq \gamma_{0} \leq 20^{\circ}$ scintillation level decreases in both $\Upsilon<<1$ and transition regions. Splashes arises in the region $\Upsilon>>1$ : at $\Upsilon=500$ $\left(k_{f}=4 \mathrm{~km}^{-1}\right)$ for $\gamma_{0}=0^{0}$ (curve 1$)$, at $\Upsilon=840\left(k_{f}=3\right.$ $\mathrm{km}^{-1}$ ) for $\gamma_{0}=10^{0}$ (curve 2), at $\Upsilon=1420\left(k_{f}=2 \mathrm{~km}^{-1}\right.$ ) for $\gamma_{0}=20^{\circ}$ (curve 3). Oscillations are revealed in a transient region (shaded area) at $\gamma_{0}=0^{0}$. The scintillation level is estimated for different anisotropy factors and orientation angle of elongated irregularities with respect to the external magnetic field. Scintillation minimums of a temporal spectrum can be calculated for different thicknesses of a plasma slab taking into account the movement of rigid irregularities and diffraction effects. 
Figures 13 and 14 show log-log plots of the phase scintillation spectrum with Fresnel oscillations for the spectrum (9), plasma irregularities are moving along the $X$ direction with the velocity $V_{x}=100 \mathrm{~meter} / \mathrm{sec}$. In this case $\alpha=20^{\circ}$. Solid line corresponds: $\xi=10\left(l_{\|}=160 \mathrm{~m}\right)$ and $\chi=100\left(l_{\perp}=1.6 \mathrm{~m}\right)$, dashed line $\xi=20\left(l_{\|}=320 \mathrm{~m}\right)$ and $\chi=60 \quad\left(l_{\perp}=5.3 \mathrm{~m}\right)$. Ordinary and extraordinary waves have first minimum at $v_{1}=0.11 \mathrm{~Hz}$, next minimums are at $v_{2}=0.15 \mathrm{~Hz}, v_{3}=0.18 \mathrm{~Hz}, v_{4}=0.21 \mathrm{~Hz}, v_{5}=0.24 \mathrm{~Hz}$; or multiplying first minimum on: $(2)^{1 / 2},(3)^{1 / 2}$ and $(4)^{1 / 2}$. Figure 14 is a log-log plots of the power spectrum of the intensity fluctuations, at $\xi=20$ and $\chi=60$. Irregularities are moving along $X$ (solid line) and $Y$ (dashed line) directions with the velocity $V_{x, y}=100 \mathrm{~meter} / \mathrm{sec}$. The location of maximums in both cases is the same: $v_{1 \max }=$ $0.07 \mathrm{~Hz}, \ldots v_{5 \max }=0.22 \mathrm{~Hz}$.

\section{Conclusions}

Second order statistical moments are calculated solving differential equation for the phase fluctuations taking into account the boundary condition, diffraction effects and polarization coefficients both of the ordinary and extraordinary waves. Numerical calculations are carried out for $3 \mathrm{MHz}$ incident electromagnetic wave using the experimental data. The variance and the correlation function are calculated for arbitrary 3D spectral function of electron density fluctuations containing both anisotropic Gaussian and power-law spectra. This spectrum of the elongated plasma irregularities contains anisotropy coefficient and the orientation angle with respect to the static external magnetic field. It is shown that $3 \mathrm{D}$ normalized correlation function oscillates for the Gaussian spectrum; power-law spectrum with the spectral index 4 becomes smooth these oscillations. Geometric parameters of plasma irregularities have a substantial influence on the ordinary and extraordinary waves. Increasing characteristic spatial scale of large-scale irregularities phase scintillation index $S_{4}$ growth tending to the saturation. Splashes are revealed for different anisotropy factor of elongated largescale irregularities varying orientation angle with respect to the lines of force of geomagnetic field. Scintillation index is calculated for small-scale irregularities using the "frozenin" assumption taking into account movement of rigid irregularities both in the principle $Y Z$ plane and in normal directions. Anisotropic Gaussian spectrum is responsible for scintillation of these irregularities. Maximum $S_{4 \max }$ is in the interval 0.2-0.3 indicating conditions of weak scatter of the radio wave by the ionospheric irregularities. The observed $S_{4}$ values are less than 0.55 corresponding to the moderate scintillation intensity, within the weak-scatter regime.

Log-log plots of the normalized scintillation level versus parameter $\Upsilon=2\left(k_{0} / k_{f}\right)^{2}$ containing Fresnel wave number are constructed for different geometric parameters characterizing electron density fluctuations. Increasing anisotropy factor $\chi$ scintillation level growth in both nonfully-developed diffraction pattern $(\Upsilon<<1)$ and in transition zone. Rising orientation angle $\gamma_{0}$ scintillation level decreases and splashes arises in fully developed scintillation region.

The log-log plots of the power spectrum of the intensity fluctuations of the ordinary and extraordinary waves scattered on a moving rigid strongly elongated small-scale plasma irregularities have the same minimums satisfying the "standard relationship"- multiplying first minimum on $(2)^{1 / 2},(3)^{1 / 2},(4)^{1 / 2} \ldots$

Comparison of the Gaussian and power-law spectra in more detail requires that the scintillation spectra be compared with both one- and two dimensional weakscattering scintillation models. Simultaneous scintillation measurements in a wide range of frequencies should be made to establish the frequency dependence of scintillation index. This provides important information about the spectrum of the irregularities, amplitude and altitude distribution of irregularities [14].

Scintillation studies are of importance not only because of their direct application in transionospheric communications, but also for the understanding of the mechanisms responsible for the generation and growth of ionospheric irregularities. Using the known geomagnetic field and the direction where the maximum scintillation is observed, one can determine the parameters of 3D anisotropy from certain statistical parameters of the phase of the scattered signal.

\section{Acknowledgements}

This work has been supported by the International Science and Technology Center (ISTC) under Grant \# G-2126 and Shota Rustaveli National Science Foundation under Grant \# FR/3/9-190/14.

\section{References}

[1] A. Ishimaru, A. Wave Propagation and Scattering in Random Media, Vol. 2, Multiple Scattering, Turbulence, Rough Surfaces and Remote Sensing, IEEE Press, Piscataway, New Jersey, USA, 1997.

[2] S.M. Rytov, Yu.A. Kravtsov, V. I. Tatarskii, Principles of Statistical Radiophysics. vol.4. Waves Propagation Through Random Media. Berlin, New York, Springer, 1989.

[3] B.N. Gershman, L.M. Erukhimov, Yu.Ya. Yashin, Wave phenomena in the Ionosphere and Space Plasma, Moscow, Nauka, 1984.

[4] V.L. Frolov, N.V. Bakhmet'eva, V.V. Belikovich, G.G. Vetrogradov, V.G. Vatrogradov, G.P. Komrakov, D.S. Kotik, N.A. Mityakov, S.V. Polyakov, V.O. Rapoport, E.N. Sergeev, E.D. Tereshchenko, A.V. Tolmacheva, V.P. Uryadov, and B.Z. Khudukon, Modification of the earth's ionosphere by high-power high-frequency radio waves Phys. Usp., 50: 315-325, 2007. 
[5] Z.-W. Xu, J. Wu, Z.-S. Wu, A survey of ionospheric effects on space-based radar, Waves in Random media 14: 189-273, 2004.

[6] Z.-S. Wu, , H.-Y. Wei, R.-K. Yang, L.-X. Guo,. Study of scintillation considering inner and outer-scales for laser beam propagation on the slant path through the atmospheric turbulence, PIER 80: 277-293, 2008

[7] G.V. Jandieri, Zh.M. Diasamidze, M.R. Diasamidze, I.G. Takidze, Second order statistical moments of the power spectrum of ionospheric scintillation Atmospheric and Oceanic Sciences 2: 90-96, 2017.

[8] G.V. Jandieri, Zh.M. Diasamidze, M.R. Diasamidze, Scintillation effects and the spatial power spectrum of scattered radio waves in the ionospheric $\mathrm{F}$ region, Journal of Advances in Physics 13: 4593-4604, 2017.

[9] G.V. Jandieri, Zh.M. Diasamidze, M.R. Diasamidze, Scintillation spectra of scattered electromagnetic waves in turbulent magnetized plasma, Journal of Basic and Applied Physics, 2: 224-234, 2013.

[10] V.L. Ginzburg, Propagation of Electromagnetic Waves in Plasma, Gordon and Beach, New York, 1961.

[11] G.V. Jandieri, A. Ishimaru, Some Peculiarities of the Spatial Power Spectrum of Scattered Electromagnetic Waves in Randomly Inhomogeneous Magnetized Plasma with Electron Density and External Magnetic Field Fluctuations, PIER B, 50: 77-95, 2013.

[12]C.L. Rufenach, Ionospheric scintillation by a random phase screen: Spectral approach, Radio Science, 2: 155-165, 1975.
[13]C.L. Rufenach, Power spectra of large scintillation signals, J. Atmos. Terr. Phys., 37: 569-572, 1975.

[14]C.L.Rufenach, Power-law wavenumber spectrum deduced from ionospheric scintillation observations, Journal of Geophysical Research, 77: 4761-4772, 1972.

[15] S.Raizada, H.S.S. Sinha, Some new features of electron density irregularities over SHAR during strong spread F. Ann. Geophysicae, 18: 141-151, 2000.

[16] S.S. Prakash, S. Pal, H. Chandra, In-situ studies of equatorial spread $\mathrm{F}$ over SHAR-steep gradients in the bottomside F-region and transitional wavelength results. J. Atmos. Terr. Phys., 53: 977-986, 1991.

[17] H.O. Vats, Survey of diffractive-refractive scintillation theory, J. Atmos. Terr. Phys., 51: 739-742, 1989.

[18] N.V. Bakhmet'eva, V.N. Bubukina, Yu.A. Ignat'ev, G.S. Bochkarev, V.A. Eremenko, V.V. Kol'tsov, I.V. Krasheninnikov, Yu.N. Cherkashin, Investigation by backscattering radar of artificial irregularities produced in ionospheric plasma heating experiments. J. Atmos. SolarTerr. Phys., 59: 2257-2263, 1997.

[19] N.D. Kvavadze, Z.L. Liadze, N.V. Mosashvili, Z.S. Sharadze, The phenomenon of F-scattering and drift of smallscale irregularities at night low latitudes F-region of an ionosphere, Geomagnetizm and Aeronomy, 28: 139-141, 1988.

[20] A.W. Wernik, C.H. Liu, Ionospheric irregularities causing scintillation of $\mathrm{GHz}$ frequency radio signals, J. Atmos. Terr. Phys., 36: 871-879, 1974.

[21] E.J. Fremouw, Geometrical control of the radio of intensity and phase scintillation indices, J. Atmos. Terr. Phys., 42: 775-782, 1980. 